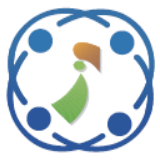

\title{
An Efficient Hierarchical 3D Mesh Segmentation Using Negative Curvature and Dihedral Angle
}

\author{
Khadija Arhid $^{1 *}$ Fatima Rafii Zakani $^{1}$ Mohcine Bouksim ${ }^{1}$ Mohamed Aboulfatah ${ }^{2}$ Taoufiq Gadi ${ }^{1}$ \\ ${ }^{1}$ Laboratory of Informatics, Imaging, and Modeling of Complex Systems (LIIMSC) \\ Faculty of Sciences and Techniques, Hassan 1st University, Settat, Morocco \\ ${ }^{2}$ Laboratory of Analysis of Systems and Treatment of Information (LASTI) \\ Faculty of Sciences and Techniques, Hassan 1st University, Settat, Morocco \\ * Corresponding author's Email: khadija.arhid@gmail.com
}

\begin{abstract}
Decomposing a 3D mesh into significant regions is considered as a fundamental process in computer graphics, since several algorithms use the segmentation results as an initial step, such as, skeleton extraction, shape retrieval, shape correspondence, and compression. In this work, we present a new segmentation algorithm using spectral clustering where the affinity matrix is constructed by combining the minimal curvature and dihedral angles to detect both concave and convex properties of each edge. Experimental results show that the proposed method outperforms some of the existing segmentation methods, which highlight the performance of our approach.
\end{abstract}

Keywords: 3D mesh, 3D segmentation, Spectral clustering, Negative curvature, Dihedral angle.

\section{Introduction}

3D mesh segmentation is considered as an important step towards mesh analysis and understanding, the reason why it has been the subject of considerable research activity over the last decade. Hence, many approaches have been extensively investigated with the development of a large number of new algorithms and methodologies [1].

The task of 3D mesh segmentation consists on detection of homogeneous regions in the mesh, either from a semantic or from a geometric point of view and the efficiency of a segmentation method is in most cases application dependent. In fact, mesh segmentation is classified into two general categories: part type which partitions the mesh into meaningful regions using both surface and volumetric attributes, and surface type that partitions the 3D model into meaningful surface parts approximated by various surface primitives.

The interest behind mesh segmentation is object recognition, the reason why Hoffman and Richards [2] proposed the theory of object recognition by parts. This theory states that humans perform decomposition at negative minima of principal curvature, which lead to a shape decomposition on convex regions. However, other researchers [3, 4] see that instead of generating cuts in concave boundaries they generate parts which correspond to convex boundaries. Such segmentation is useful for applications such as collision detection.

In this paper, we propose a segmentation method based on spectral clustering and both minimal curvatures and dihedral angles for the construction of the affinity matrix. Knowing that affinity matrix encodes the similarity between two adjacent faces and according to the minima rule, the significant cuts should be across deep concavity, which is not always true since in some cases negative minimum curvatures failed to find the entire best boundaries. For this reason, we propose to combine the minimal curvatures and dihedral angles to catch respectively concave and convex edges in order to accordingly affect a weight for each edge in the adjacency matrix.

The rest of this paper is organized as follows: In section 2 , we briefly present an overview of some 
segmentation algorithms. Section 3, outlines the detailed of the proposed approach while section 4 is dedicated to the experimental results showing the efficiency of our segmentation method. Finally, we summarize our work with a conclusion.

\section{Related work}

In the past few years, a great number of 3D mesh segmentation algorithms have been proposed in the literature. Each segmentation method is based on a core methodology which belongs to a type of mesh segmentation methods. In this overview of mesh segmentation algorithms, we will present three categories: Region growing, Clustering, and spectral analysis.

\subsection{Clustering methods}

The clustering consists of assigning each face or vertex to the nearby segment based on primitives (plane, sphere, cylinder, etc.). The core of this method is regrouping similar mesh elements based on the calculation of the distance of each (face or vertex) to the cluster centroid. These seed points are choosing by maximizing the total pairwise distance between them. Users usually specify the number of clusters. In this category, segmentation is achieved either by an iterative clustering using k-means algorithm or by direct techniques where the initial assignment will determine the final segmentation. The main drawback of this technique is its sensitivity to random choice of the initial cluster centers; and consequently, if the initial selection is bad, the resulted segmentation is not guaranteed to be well. Garland et al [5] propose a hierarchical clustering method, the clusters in this work are chosen so that they can be approximated with planar elements. This method is based on pairwise cluster joining where each pair of clusters are assigned a cost of merging them to one cluster, and the lowest cost pair is combined. This cost is based on planarity, orientation bias, and shape bias measures and for detecting the planarity of clusters during clustering, a dual quadric error metric was introduced. This algorithm is suitable for radiosity or simplification, and less applicable for applications seeking the significant components. Yamauchi et al. [6] proposed a segmentation method based on clustering mesh normals using mean shift algorithm. The proposed method is robust to local noise however it this method leads to over segment a mesh into more parts than desired. Shlafman et al. [7] propose an iterative clustering algorithm using k-means to partition meshes into meaningful parts. A $\mathrm{K}$ seed faces were determined to represent patch centers by maximizing the pairwise distances between them. Then, all faces are assigned to their nearest seed faces, and the cluster centroids are recalculated by minimizing the sum of distances between the faces belonging to the segment and the representative. This process is repeated until convergence. The used pairwise distance is a weighted sum of the angle between the faces and the geodesic distance of their barycenters.

\subsection{Region growing}

This technique is one of the simplest segmentation approaches. It starts by specifying seed elements and grows these seeds by progressively merging adjacent faces or vertices. The results of this kind of methods depend on two criterions: the selection of seeds which are in most cases local minima and the growing criteria which determine if an element can be appended to an existing segment or not. The major drawbacks of this approach are the selection of the exact seed elements since they do not change and it requires a post-processing merging stage to overcome the over-segmentation that consume more running time. Kalvin and Taylor [8] propose a Superfaces algorithm based on region growing technique. The procedure of face clustering is done by partitioning the surface into a set of individual face clusters, or "super faces". This algorithm was founded on growing a single cluster around a seed face selected randomly, merging new faces into the current cluster until this later exceeded a planarity threshold. Lavoué et al. [9] proposed a segmentation algorithm using the absolute values of the maximum and minimum principal curvature of vertices to construct regions with a similar geometric criterion. Vertices are firstly clustered using k-means algorithm based on their principal curvature, then a process of region growing is done to connect adjacent faces of the same cluster which do not share a sharp edge, creating clusters of similar curvature. Zhang et al. [10] also proposed a segmentation method based on a region growing technique which decomposes meshes into separate regions based on Gaussian curvature analysis. As a first step, an estimation of vertices Gaussian curvature is done to detect boundaries composed of vertices with highly negative curvature then a growth rule based on component labeling process is performed to enlarge non-boundary vertices into regions. A segmentation method based on region growing technique is also proposed by Zuckerberger et al. [3] which start by extracting the dual graph of the mesh, and then the algorithm selects a seed element randomly and 
continues to collect nodes or faces which form a convex region. When the convexity is violated, the process of computing new segment is launched from an unvisited node. The main problem with this method is over-segmentation which the authors propose to outfit by merging smaller patches to adjacent larger ones.

\section{3 spectral analysis}

Spectral analysis is one of the well-known segmentation technique since it underlines global shape properties using the local connectivity. These methods rely on eigenvalues and eigenvectors of the adjacency matrix constructed for the graph representing the input mesh. This approach is considered as one of the best and most used technique since it provides the best semantic results. However, the performance of these methods is proportional to the features edges and the weight assigned to each edge in the similarity matrix. Spectral clustering was firstly introduced to segment 3D meshes by Liu and Zhang et al. [11] the authors create an adjacency matrix which encodes distances between mesh faces utilizing a combination of angular and geodesic distances to don't group faces separated by deep concave regions in the same partition. Then a spectral embedding of the mesh faces is done using the eigenvector of the graph Laplacian. Later, Zhang and Liu et al. [12] propose a segmentation method based on a recursive 2-way spectral clustering using the same affinity matrix as in Liu et al. [11] which provide information for a visually meaningful segmentation. Then the first two largest eigenvectors are computed which are used in a line search algorithm to find the most salient cut to bipartition the mesh. This process is repeated until the segmentation of the mesh. More recently, Chahhou et al. [13] propose a method using spectral clustering. In this approach, the affinity matrix is constructed using the minima rule to encode the connectivity of the mesh after that the spectral clustering is used to find the best cuts through the convex area.

\section{Our Approach}

In last few years, spectral clustering has been considered as one of the important and efficient clustering algorithms. It has many major advantages like its generality, efficiency, its rich theoretical foundation and it can be applied to any data. In computer vision, spectral clustering algorithms have been extensively exploited for segmenting 3D meshes $[11,13-16]$. The most of existing spectral clustering methods for mesh segmentation usually employ the minima rules [2] to construct the adjacency matrix; this later is the most important and decisive component of spectral clustering system because it encodes structural information of the mesh, which reflects how faces or vertices will be grouped. In this paper, we propose a new 3D mesh segmentation algorithm based on the dihedral angles and negative curvatures for generating the adjacency matrix and the Normalized Cheeger Cuts as a criterion of partitioning.

\subsection{The affinity matrix and graph cut criteria}

Let consider $G(V, E, W)$ as an undirected, weighted graph, where $V$ represents a set of vertices $\left\{v_{1}, v_{2}, \ldots, v_{3}\right\}, E$ is the set of edges and $w_{i j}$ represent the weight for the edge connecting two vertices $v_{i}$ and $v_{j}$. The affinity matrix is defined as follows $[17$, 18]:

$$
W= \begin{cases}w_{i j} & \text { if } v_{i} \text { and } v_{j} \text { are adjacent } \\ 0 \quad \text { otherwise }\end{cases}
$$

Where $v_{i}$ represent the $i^{\text {th }}$ vertex of the given graph.

Taking the fact that a 3D mesh can be represented as a graph, 3D mesh segmentation can be rephrased in the language of graphs in which the goal is to find a partition of the graph such that the edges between different clusters have a very low weight, while the edges in the same cluster have a height similarity.

In addition to the adjacency matrix, a number of other components represent a fundamental role in spectral clustering. One such is the Laplacian matrix that aims to represent graphs in a certain way. Spectral graph theory is the study of these matrices which include much valuable information about the graphs. Let $L$ be the Laplacian of the given graph; $L$ is computed as $[17,18]$ :

- Unnormalized graph Laplacian: $L=D-W$.

- Normalized graph Laplacian: $L=I-D^{-1} W$.

Where $D$ and $I$ represent respectively the degree and the identity matrices. The goal is to cluster the mesh into dissimilar regions $C_{1}$ and $C_{2}$ by minimizing the following function $[17,18]$ :

$$
\operatorname{Cut}\left(C_{1}, C_{2}\right)=\sum_{i \in C_{1}, j \in C_{2}} w_{i j}
$$

The minimum cut criterion is a suitable solution to illustrate the idea of the min-cut graph; however, it does not lead to reasonable results if the connected components are not balanced and consequently 
finding small clusters. The Normalized cut [19] and the Ratio Cut [20] functions are proposed to alleviate this problem and overcome the drawback of minimum cut partitioning and create balanced partitions:

$$
\begin{aligned}
& \operatorname{RCut}\left(C_{1}, C_{2}\right)=\frac{\operatorname{Cut}\left(C_{1}, C_{2}\right)}{\left|C_{1}\right|}+\frac{\operatorname{Cut}\left(C_{1}, C_{2}\right)}{\left|C_{2}\right|} \\
& \operatorname{NCut}\left(C_{1}, C_{2}\right)=\frac{\operatorname{Cut}\left(C_{1}, C_{2}\right)}{\operatorname{vol}\left(C_{1}\right)}+\frac{\operatorname{Cut}\left(C_{1}, C_{2}\right)}{\operatorname{vol}\left(C_{2}\right)}
\end{aligned}
$$

Where $|C|$ is the cardinality of the set $C$ while $\operatorname{vol}(C)$ representing the volume of the set $C$ calculated as the sum of the weights of all edges attached in $C$.

Others formulations of clustering that aim to balance the min Cut criterion are defined as:

- The Ratio Cheeger Cut:

$$
\operatorname{RCC}\left(C_{1}, C_{2}\right)=\frac{\operatorname{Cut}\left(C_{1}, C_{2}\right)}{\min \left(\left|C_{1}\right|,\left|C_{2}\right|\right)}
$$

- The Normalized Cheeger Cut:

$$
\operatorname{NCC}\left(C_{1}, C_{2}\right)=\frac{\operatorname{Cut}\left(C_{1}, C_{2}\right)}{\min \left(\operatorname{vol}\left(C_{1}\right), \operatorname{vol}\left(C_{2}\right)\right)}
$$

It is known from graph theory that the optimal solution to the Laplacian graph-partitioning problem is given by the eigenvector to the second smallest eigenvalue of $L$. the eigenvalues can be found by solving the linear equation system $L v=\lambda D v[13,17]$.

According to [13], the Normalized Cheeger Cuts (NCC) tends to find the best partitioning of the mesh into two different part by minimizing the $\operatorname{Cut}\left(C_{1}, C_{2}\right)$ and maximizing the ratio of the volume of the clusters. For this reason, we opt to use the $N C C$ that provides best semantic results.

\subsection{The affinity matrix and graph cut criteria}

Curvature estimation is an essential mechanism for analyzing and characterizing a surface's behavior. For each vertex on a mesh, there are two principal curvatures denoted $k_{\min }$ and $k_{\max }$ which represent the minimum and the maximum curvatures [21]. The minima rule [2] affirms that human perceptual system tends to cut meshes through negative minimum principal curvatures. Based on this later, we tend to find boundaries having deep concavity, in which the faces separated by these edges are less likely to be grouped together in the same cluster. However, the major problem encountered in some cases is when the significant cut should be obtained across the convex zones. For example, consider the shoulder joint of a human, or a cup object (Fig. 1). At this point, the negative minimum curvatures are not guaranteed to find the entire best boundaries. To get over this problem, we propose to combine the dihedral angle between two faces which represent a geometric information that succeeds to detect the convex regions and the negative minimum principal curvatures to construct a weight for each edge.

In our proposed approach, the main idea is to include two prominent feature edges. Firstly, the principal curvatures are computed for each face using the approach proposed in [21]. Then we define the first candidate boundaries by considering only the edges separating faces having the maximum of minimum principal curvatures of all $n$ neighbors is smaller to a certain threshold $\mu, \max \left(k_{\min }^{1}, k_{\min }^{2}, \ldots\right.$, $\left.k_{\text {min }}^{n}\right) \leq \mu$. It is important to note; that we consider all adjacent faces into account to catch more appropriate information of the current face and avoid select false minima. In [14] we find that a slight difference to zero in choosing negative curvature give excellent result and consequently, by experiments we fixed $\mu=-5$ in our case.

Then, using the dihedral angle, we define the feature edges that not have been detected in the first step even if they also represent significant boundaries. It is a differential geometric property widely used to partitioning $3 \mathrm{D}$ mesh $[1,3,22]$. The dihedral angle between two faces $f_{i}$ and $f_{j}$ is calculated by:

$$
\theta\left(f_{i}, f_{j}\right)=\operatorname{arcos}\left(\frac{\operatorname{dot}\left(\vec{N}_{i}, \overrightarrow{N_{j}}\right)}{\left|\overrightarrow{N_{i}}\right| \times\left|\overrightarrow{N_{j}}\right|}\right)
$$

Where $\vec{N}_{l}$ is the normal vector of the face $f_{i}$ and $|\vec{N}|$ represent the norm of the vector $\vec{N}, \operatorname{dot}\left(\overrightarrow{N_{l}}, \overrightarrow{N_{J}}\right)$ is the scaler product between two vectors $\overrightarrow{N_{l}}$ and $\overrightarrow{N_{J}}$.

An edge is considered as a convex feature boundary if:

$$
\theta_{\min } \leq \theta\left(f_{i},\left\{f_{j}\right\}\right) \leq \theta_{\max }
$$

Where $\theta\left(f_{i},\left\{f_{j}\right\}\right)$ is the average of the dihedral angles between the face $f_{i}$ and their neighbors $\left\{f_{j}\right\}, \theta_{\min }$ and 
$\theta_{\max }$ are the minimum and maximum thresholds, by experiments we have found $\theta_{\min }=9^{\circ}$ and $\theta_{\max }=50^{\circ}$ produce good results for general case.

In the last step, we add a post-processing stage in which small boundaries are truncated using the area of faces in the contour as a criterion to decrease the overall the false feature edges. Then, we construct our adjacency matrix as follows:

$$
W(i, j)=\left\{\begin{array}{ll}
0.1 \quad \text { if } f_{i} \text { and } f_{j} \in \text { concave regions } \\
0.2 \quad \begin{array}{l}
\text { if } f_{i} \text { and } f_{j} \in \text { convex regions } \\
1 \quad
\end{array} \quad \begin{array}{l}
\text { if } f_{i} \text { and } f_{j} \text { are adjacent } \\
\& \& \notin \text { concave regions } \\
\& \& \notin \text { convex regions } \\
0 \quad \text { otherwize }
\end{array}
\end{array}\right\}
$$

Where $f_{i}$ is the $i^{\text {th }}$ face of the given graph and $W(i, j)$ represent the weight of the edge connecting two adjacent faces $f_{i}$ and $f_{j}$.

The Fig. 1 shows the obtained minima using the previous steps; the blue faces represent the set of minima produced using the negative curvatures while the green ones illustrate the minima achieved with the dihedral angles. Therefore, we must, ensure that the weights associated to the edges of these regions are sufficiently small to favor cuts in these areas instead to the other possible cuts, we have fixed these values to 0.1 and 0.2 experimentally since they provide the best semantic segmentation.

After generating the adjacency matrix and all weights are assigned to the edges of the graph according to a combination between negative principal curvatures and dihedral angles. The 3D mesh is partitioned through the Normalized Cheeger Cut criterion $(N C C)$. The recursive partitioning based on two-way Spectral Clustering is done until the desired $\mathrm{k}$ clusters are achieved.

\section{Experimental study}

In the following, we investigate and evaluate our proposed approach through several experiments on the Benchmark of Princeton that includes 380 3D models classified into 19 classes and accompanied with their ground truth and automatic segmentations[23]. The performance of our approach is evaluated using different assessment metrics of proposed in the literature.

In the first experiment, we highlight the benefit of the proposed method in qualitative point of view by showing the visual results for each category.

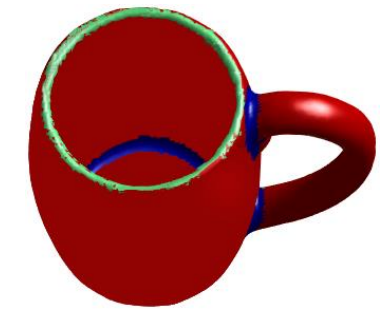

Figure.1 The obtained minima using our approach

As can be observed in Fig. 2, for the vast majority of classes, the segmentations obtained running our algorithm are more challenging and having a high similarity to the ground truth segmentation got by human perception and consequently, our approach

represents a suitable solution for producing useful segmentations.

The second test aims to evaluate the proposed method using two evaluation measures the Adaptive Entropy Increment [24] and the Weighted Levenshtein distance [25] proposed by our research team, in order to quantify the quality of our segmentation approach. Then we perform a comparison with four well-known segmentation algorithms, which are K-Means (KM) [7], Fitting Primitives (FP) [26], Normalized Cuts (NC) and Randomized Cuts (RC) [27]. Fig. 3, Fig. 4 and Fig. 5 illustrate the obtained scores of the compared algorithms for each category. Besides, Fig. 6 shows a visual comparison of some extracted models from the evaluated data set. As can be deduced from the qualitative and quantitative comparison, in the most cases, our approach achieves law dissimilarity scores very close to one of the best segmentation algorithms in the literature, which is the Randomized Cuts and outperform the three other algorithms.

Moreover, we have tested the performance of our approach on the data set of Benhabiles et al. Benchmark with the 3D Normalized Probabilistic Rand Index (3DNPRI)[28]. The obtained results are compared to some methodologies, which are MVBB[29], Plumber[30], Curvature Classification (CC)[9], Topology Driven (TD)[31], Fitting Primitives (FP)[32], Shape Diameter (SD)[33], Heterogeneous Graphs (HG) [14] and Boundary Learning (BL)[34]. Fig. 7 and Table 1 illustrate the comparative results produced by the evaluated algorithms. Note that the obtained results for the others algorithms are retrieved from the paper of Theologou et al. [14] and the values represent the similarity scores. Our approach gives a high similarity scores compared to the ground truth segmentations and located after the Boundary learning method, which is based on a learning step. 


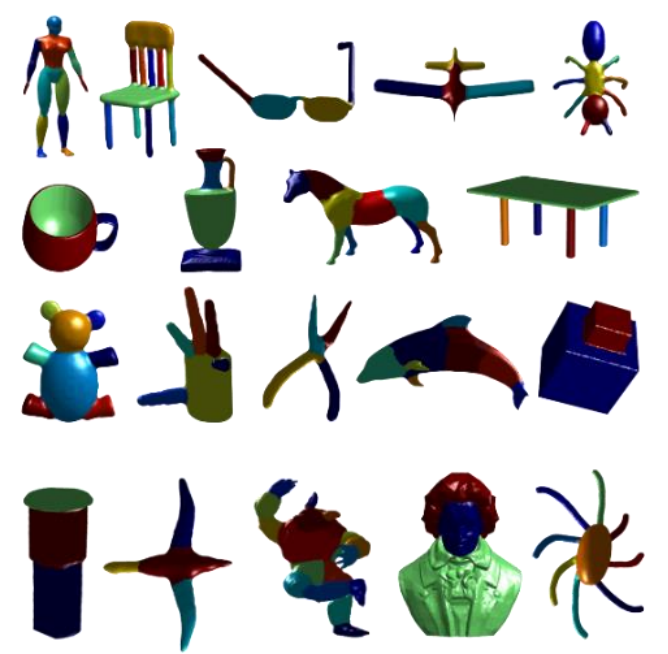

Figure.2 The obtained segmentations for each category using our approach

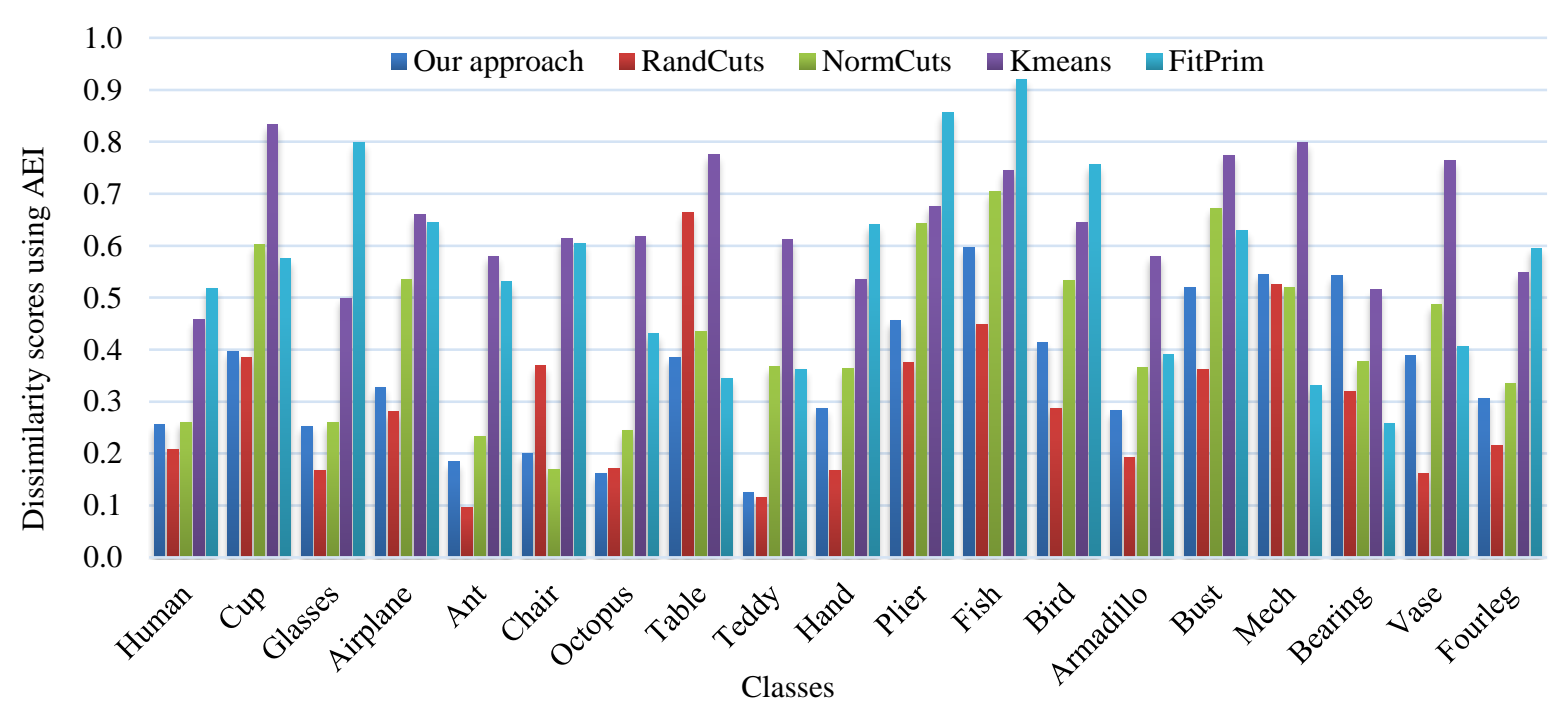

Figure. 3 The dissimilarity scores using the Adaptive entropy increment [24] for the five compared algorithms

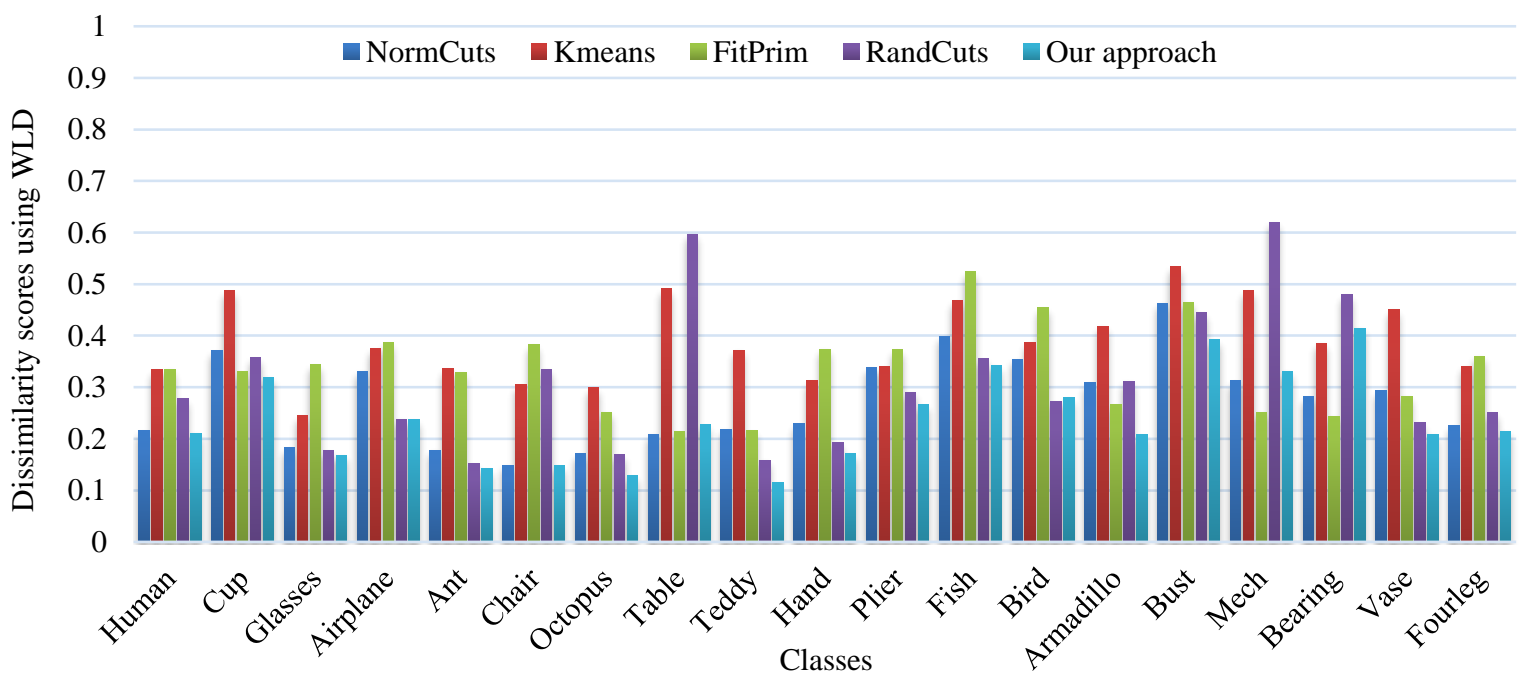

Figure. 4 The dissimilarity scores using the Weighted Levenshtein Distance [25] for the five compared algorithms 


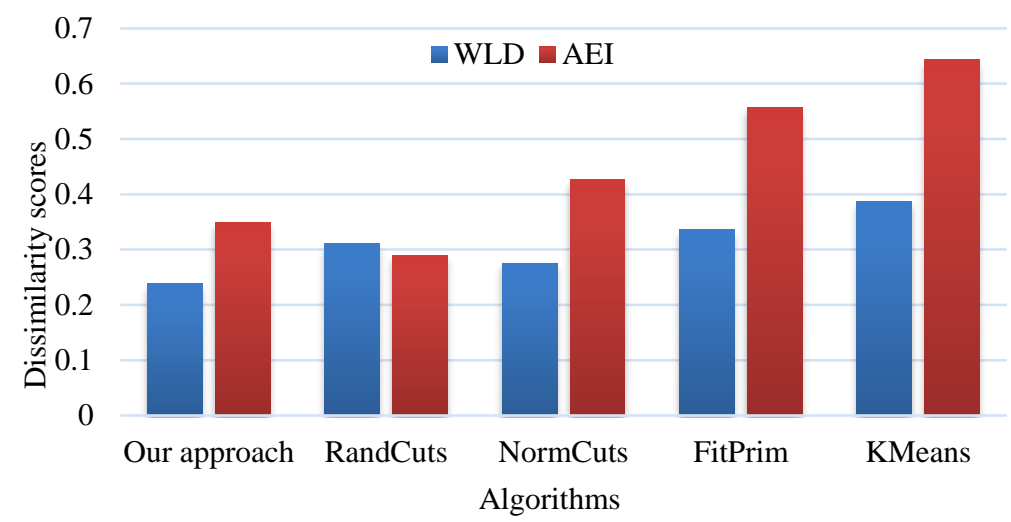

Figure. 5 The average results of the compared algorithms on the whole dataset using the Weighted Levenshtein Distance [25](WLD) and the Adaptive entropy increment (AEI)[24]

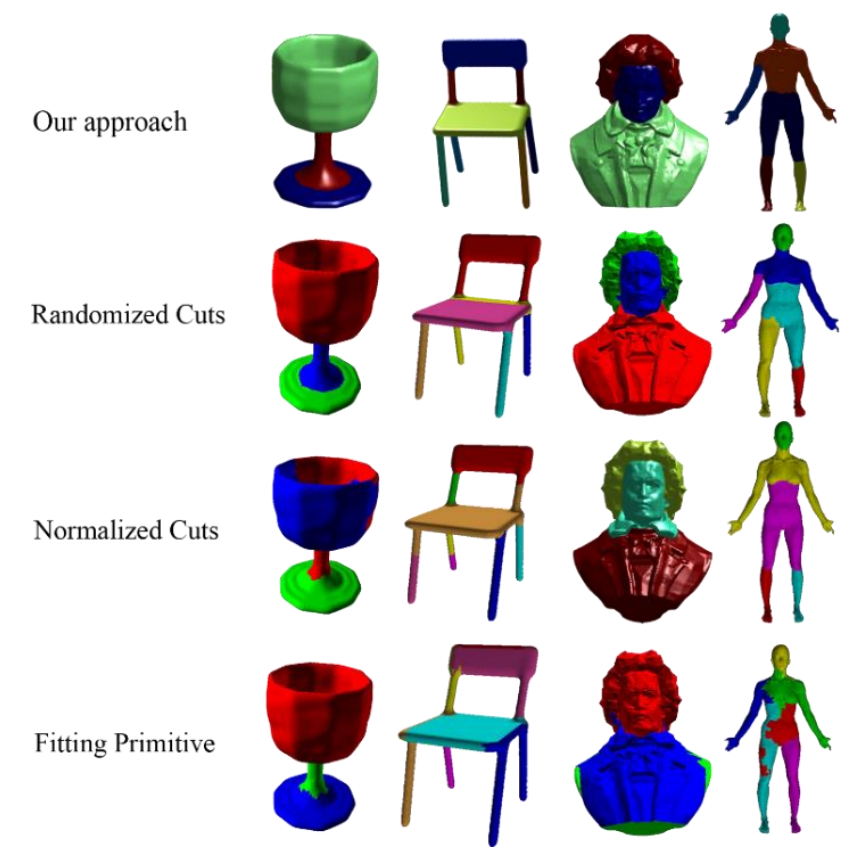

Figure. 6 A visual comparison of some extracted models from the evaluated data set using the evaluated algorithms

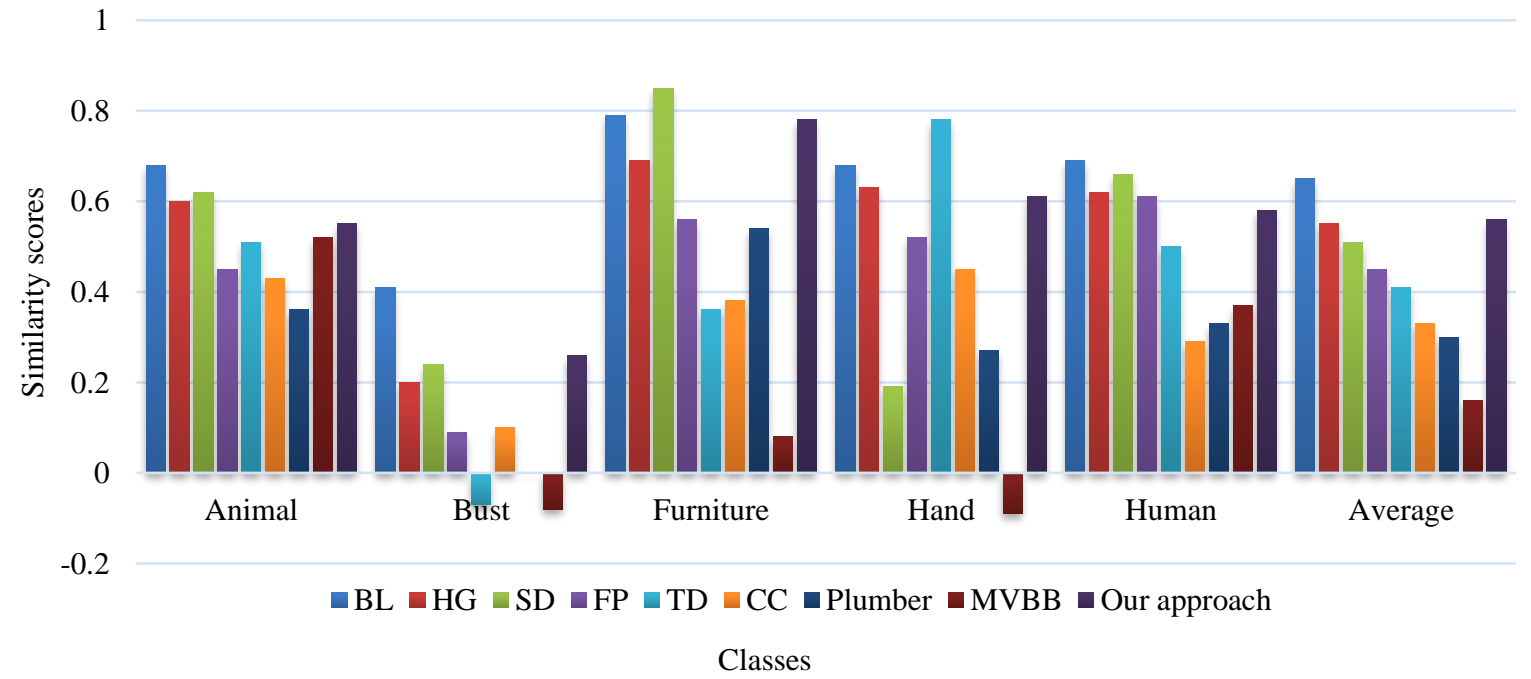

Figure. 7 A comparative evaluation of our approach to eight algorithms using the 3D Normalized Probabilistic Rand Index (3DNPRI)[28]. 
Table 1. The comparative results produced by the evaluated algorithms using the 3D Normalized Probabilistic Rand Index (3DNPRI)[28]

\begin{tabular}{|c|c|c|c|c|c|c|}
\hline Method & Animal & Bust & Furniture & Hand & Human & Average \\
\hline BL[34] & 0,68 & 0,41 & 0,79 & 0,68 & 0,69 & 0,65 \\
\hline HG[14] & 0,6 & 0,2 & 0,69 & 0,63 & 0,62 & 0,55 \\
\hline SD[33] & 0,62 & 0,24 & 0,85 & 0,19 & 0,66 & 0,51 \\
\hline FP[26] & 0,45 & 0,09 & 0,56 & 0,52 & 0,61 & 0,45 \\
\hline TD[31] & 0,51 & $-0,07$ & 0,36 & 0,78 & 0,5 & 0,41 \\
\hline CC[9] & 0,43 & 0,1 & 0,38 & 0,45 & 0,29 & 0,33 \\
\hline Plumber[30] & 0,36 & 0 & 0,54 & 0,27 & 0,33 & 0,3 \\
\hline MVBB[29] & 0,52 & $-0,08$ & 0,08 & $-0,09$ & 0,37 & 0,16 \\
\hline Our approach & 0,55 & 0,26 & 0,78 & 0,61 & 0,58 & 0,56 \\
\hline
\end{tabular}

Finally, in Fig. 8, we generate using our proposed method several segmentations of cup, human and bird models with different numbers of segments to show how our approach behaves toward hierarchical desired segmentation. From the produced results, our approach has a good behavior in generating semantic hierarchical segmentation. A mesh can be recursively decomposed reasonably using the proposed algorithm until the desired number of clusters.

\section{Conclusion}

3D mesh segmentation is a fundamental element in many applications in computer vision. Due to its importance, the developments of 3D segmentation methods are in continuous extension. Spectral clustering has been considered as one of the important and efficient segmentation algorithms. In this paper, we have proposed a new algorithm for 3D mesh segmentation based on spectral clustering. The proposed approach is based on the minimum negative curvature and the dihedral angle to find out all feature edges and then to construct the adjacency matrix. Next, the Normalized Cheeger Cut was used to cluster the similar faces in the same patch. An experimental study was performed to illustrate the performance of the proposed method. These results demonstrate the power of taking into consideration both concave and convex edge in the construction of the affinity matrix to decompose an object into visual parts. As a future work, we will try to use the same concept to propose a segmentation method based on the detection of boundaries.

\section{Acknowledgments}

We would like to thank the anonymous reviewers for their valuable comments, which help to improve the quality of the paper. We also like to thank Zhenbao Liu and Panagiotis Theologou for helping us with the source code for their methods respectively AEI and HSEG. Finally, we thank Xiaobai Chen and Halim Benhabiles for providing each one his Segmentation Benchmark, which makes it easy for us to validate the proposed segmentation method.

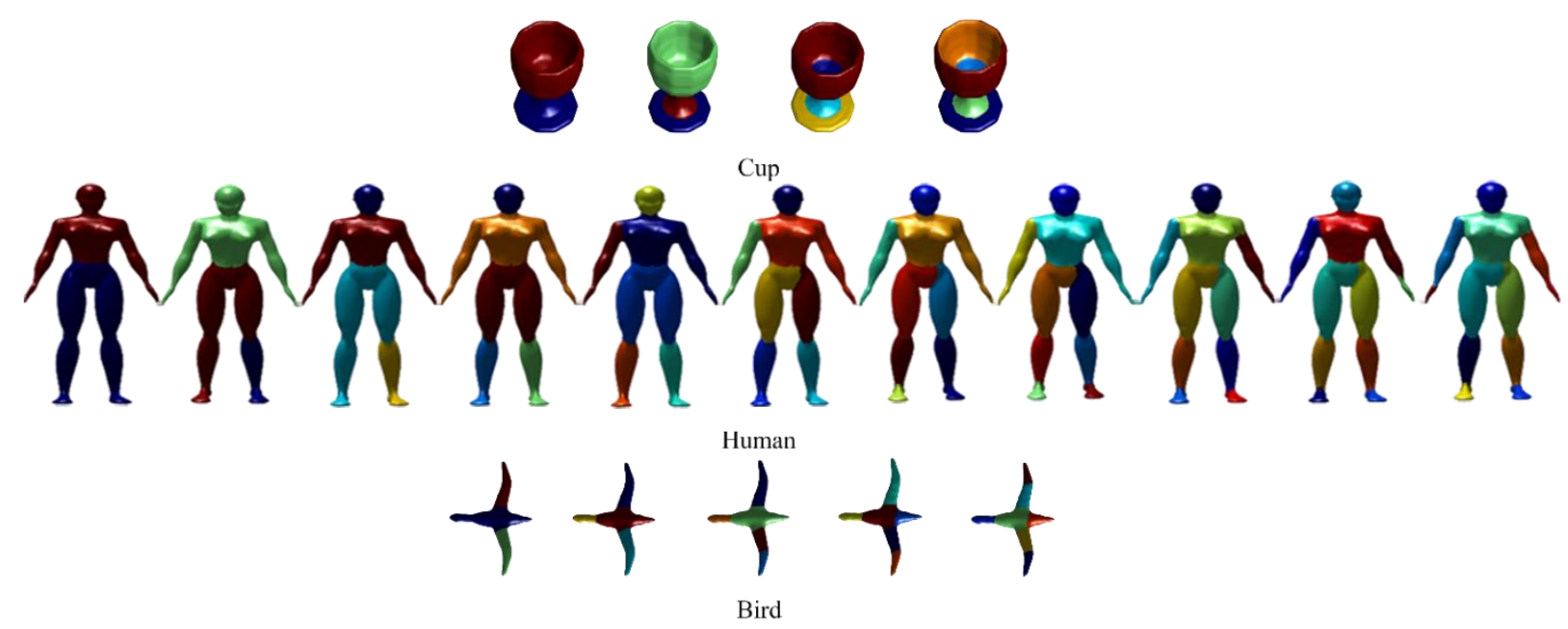

Figure. 8 Hierarchical segmentations with different number of segments for three objects: (a) Cup, (b) Human, (c) Bird International Journal of Intelligent Engineering and Systems, Vol.10, No.5, 2017

DOI: $10.22266 /$ ijies2017.1031.16 


\section{References}

[1] P. Theologou, I. Pratikakis, and T. Theoharis, "A comprehensive overview of methodologies and performance evaluation frameworks", Computer Vision and Image Understanding, Vol.135, pp.49-82, 2015.

[2] D. D. Hoffman and W. A. Richards, "Parts of recognition", Cognition, Vol.18, No.1-3 pp.65-96, 1984.

[3] E. Zuckerberger, A. Tal, and S. Shlafman, "Polyhedral surface decomposition with applications", Computers \& Graphics., Vol.26, No.5, pp.733-743, 2002.

[4] B. Chazelle and L. Palios, "Decomposition Algorithms in Geometry," Algebraic Geometry and its Applications, 27 , pp. 419447, 1994.

[5] M. Garland, A. Willmott, and P. S. Heckbert, "Hierarchical face clustering on polygonal surfaces," In: Proc. of symposium on Interactive $3 D$ graphics., New York, USA, pp.49-58, 2001.

[6] H. Yamauchi, S. Lee, Y. Lee, Y. Ohtake, A. Belyaev, and H. P. Seidel, "Feature sensitive mesh segmentation with mean shift," In: Proc. of International Conference on Shape Modeling and Applications, Cambridge, USA, pp.236-243, 2005.

[7] S. Shlafman, A. Tal, and S. Katz "Metamorphosis of Polyhedral Surfaces using Decomposition", Computer Graphics Forum, Vol.21, No.3, pp.219-228, 2002.

[8] A. D. Kalvin and R. H. Taylor, "Superfaces: Polygonal mesh simplification with bounded error", IEEE Computer Graphics and Applications,Vol.16,No.3, pp.64-77, 1996.

[9] G. Lavoué, F. Dupont, and A. Baskurt, “A new CAD mesh segmentation method, based on curvature tensor analysis", ComputerAided Design, Vol.37, No.10, pp.975-987, 2005.

[10] Y. Zhang, J. Paik, A. Koschan, M. A. Abidi, and D. Gorsich, "Simple and efficient algorithm for part decomposition of 3-D triangulated models based on curvature analysis" In: Proc. of International Conference on Image Processing, Rochester, USA, pp.III-273-III-276, 2002.

[11] R. Liu and H. Zhang, "Segmentation of 3D Meshes through Spectral Clustering", In: Proc. of the Computer Graphics and Applications, 12th Pacific Conference, Washington, USA, pp.298-305, 2004.
[12] H. Zhang, and R. Liu, "Mesh Segmentation via Recursive and Visually Salient Spectral Cuts" In: Proc. of Vision, Modeling, and Visualization, pp. 429-436, 2005.

[13] M. Chahhou, L. Moumoun, M. El Far, and T. Gadi, "Segmentation of 3D Meshes Using pSpectral Clustering", IEEE Transactions on Pattern Analysis and Machine Intelligence., Vol. 36, No. 8, pp. 1687-1693, 2014.

[14]P. Theologou, I. Pratikakis, S. Member, and T. Theoharis, "Unsupervised Spectral Mesh Segmentation Driven by Heterogeneous Graphs", IEEE Transactions on Pattern Analysis and Machine Intelligence, Vol.39, No.2, pp.397-410, 2017.

[15] H. Zhang, O. Van Kaick and R. Dyer, "Spectral Methods for Mesh Processing and Analysis" In: Proc. of Eurographics, Prague, Czech Republic, pp.1-22, 2007.

[16] L. Paulhac, V. T. Ta, and R. Mégret, "Relaxed Cheeger Cut for image segmentation" In: Proc. of the 21st International Conference on Pattern Recognition (ICPR), Tsukuba, Japan, pp. 3321-3324, 2012.

[17] T. Bühler and M. Hein, "Spectral Clustering based on the graph p -Laplacian", In: Proc. of the 26th Annual International Conference on Machine Learning, Montreal, Quebec, Canada, pp. 81-88, 2009.

[18] A. Ng, M. Jordan, and Y. Weiss, "On Spectral Clustering: Analysis and an algorithm.", Advances in neural information precessing systems, Vol. 14, pp. 849-856, 2001.

[19] J. Shi and J. Malik, "Normalized Cuts and Image Segmentation", IEEE Transactions on Pattern Analysis and Machine Intelligence Vol.22, No.8, pp. 888-905, 2000.

[20] L. Hagen and A. Kahng, "Fast spectral methods for ratio cut partitioning and clustering", In: Proc. IEEE International Conference on Computer-Aided Design Digest of Technical Papers, Santa Clara, CA, USA, pp. 10-13, 1991.

[21] D. Chen-shi and W. Guo-zhao, "Curvatures estimation on triangular mesh", Journal of Zhejiang University Science, Vol.6, No.1, pp. 128-136, 2005.

[22] Y. Fei, Z. Fan, W. Ruo-mei, L. Li, and L. Xiao-nan, "A fast and efficient mesh segmentation method based on", Applied Mathematics-A Journal of Chinese 
Universities, Vol.29, No.4, pp.468-480, 2014.

[23] X. Chen, A. Golovinskiy, and T. Funkhouser, "A benchmark for 3D mesh segmentation" ACM Transactions on Graphics, Vol. 28, No. 3, pp. 73:1-73:12, 2009.

[24] Z. Liu, S. Tang, S. Bu, and H. Zhang, "New evaluation metrics for mesh segmentation" Computers \& Graphics., Vol.37, No.6, pp. 553-564, 2013.

[25] F. R. Zakani, K. Arhid, M. Bouksim, M. Aboulfatah, and T. Gadi, "A New Evaluation Method for Mesh Segmentation Based on the Levenshtein Distance", International Review on Computers and Software (IRECOS) Vol. 11, No.12, pp. 1117-1126, 2016.

[26] M. Attene, B. Falcidieno, and M. Spagnuolo, "Hierarchical mesh segmentation based on fitting primitives", Visual Computer: International Journal of Computer Graphics, Vol.22, No.3, pp. 181-193, 2006.

[27] A. Golovinskiy and T. Funkhouser, "Randomized cuts for 3D mesh analysis", ACM Transactions on Graphics, Vol.27, No.5, pp.145:1-145:12, 2008.

[28] H. Benhabiles, J.-P. Vandeborre, G. Lavoué, and M. Daoudi, "A comparative study of existing metrics for 3D-mesh segmentation evaluation", The Visual Computer, Vol.26, No.12, pp.1451-1466, 2010.

[29] K. Huebner, S. Ruthotto, and D. Kragic, "Minimum Volume Bounding Box Decomposition for Shape Approximation in
Robot Grasping" In: Proc. of IEEE International Conference on Robotics and Automation, Pasadena, USA, pp.1628-1633, 2008.

[30] Mortara, M., G. Patanè, M. Spagnuolo, B. Falcidieno, and J. Rossignac, "Plumber: A Method for a Multi-Scale Decomposition of 3D Shapes into Tubular Primitives and Bodies", In Proc. of the ninth ACM symposium on Solid modeling and applications, Genoa, Italy, 339-344, 2004.

[31] J. Tierny, J. Vandeborre, and M. Daoudi, "Topology driven 3D mesh hierarchical segmentation", In: Proc. of IEEE International Conference Shape Modeling and Applications, Lyon, France, pp. 215-220, 2007.

[32] M. Attene, B. Falcidieno, and M. Spagnuolo, "Hierarchical mesh segmentation based on fitting primitives", Visual Computer: International Journal of Computer Graphics, Vol. 22, No. 3, pp. 181-193, 2006.

[33] L. Shapira, A. Shamir, and D. Cohen, "Consistent Mesh Partitioning and Skeletonisation using the Shape Diameter Function", Visual Computer, Vol. 24, No. 4, pp. 249-259, 2008.

[34] H. Benhabiles, G. Lavoúe, J. P. Vandeborre, and M. Daoudi, "Learning boundary edges for 3D-mesh segmentation", Computer Graphics Forum, Vol. 30, no. 8, pp. 2170-2182, 2011. 